\title{
Population-Based Screening for CKD
}

\author{
Neil R. Powe, MD, MPH, MBA $1,2,3,4$ and L. Ebony Boulware, MD, MPH ${ }^{1,2,4}$ \\ 1 Department of Medicine, Johns Hopkins University School of Medicine, Baltimore, MD \\ 2 Department of Epidemiology, Johns Hopkins Bloomberg School of Public Health, Baltimore, MD \\ 3 Department of Health Policy and Management, Johns Hopkins Bloomberg School of Public Health, \\ Baltimore, $M D$ \\ 4 Welch Center for Prevention, Epidemiology and Clinical Research, Johns Hopkins Medical Institutions, \\ Baltimore, $M D$
}

\begin{abstract}
Organizations both in the U.S. and globally have advocated for screening for chronic kidney disease with a urine test for proteinuria followed by subsequent testing with creatinine-based, eGFR determinations. Screening for a disease, such as chronic kidney disease, can have value, but a decision to screen is not a trivial decision. Criteria must be met in order to balance the aggregate benefits with the risks and costs of a screening test. We discuss how screening for chronic kidney disease meets many of these criteria, but also how the populations to whom it is applied must be targeted and the strategies for testing must be clearly defined.
\end{abstract}

Chronic kidney disease (CKD) [EF1]has emerged as an important national and worldwide public health problem because of its prevalence and potential consequences for shortened length and quality of life. One strategy to address CKD is to identify the disease early through screening programs. But screening programs are controversial having been both praised and criticized by different advocates. Several national and international organizations have made recommendations advocating routine screening for CKD, but details regarding recommended approaches to screening vary (1-6). A decision to screen is not trivial and should be based on a variety of considerations, including the potential of screening to improve health and the best scientific evidence regarding the utility of screening under differing circumstances. In this paper, we discuss the principles of screening, what might make CKD conducive to screening, evidence for and against screening, and global approaches to CKD screening.

\section{Definition of Screening}

Screening for a disease is an activity whereby persons in a defined population who are not aware of disease are tested to identify the disease. The goal of screening is to reduce the risk of progression of a disease and to reduce its complications. Hence, effective strategies to intervene and ameliorate the disease should be available. In its purest form, screening is used

Correspondence and reprint requests: Neil R. Powe, MD, Welch Center for Prevention, Epidemiology and Clinical Research, Johns Hopkins Medical Institutions, 2024 E. Monument St., Suite 2-600, Baltimore, MD 21205. Phone: 410-955-6953; fax: 410-955-0476; email: E-mail: npowe@jhmi.edu.

Financial Disclosure: None.

Publisher's Disclaimer: This is a PDF file of an unedited manuscript that has been accepted for publication. As a service to our customers we are providing this early version of the manuscript. The manuscript will undergo copyediting, typesetting, and review of the resulting proof before it is published in its final citable form. Please note that during the production process errors may be discovered which could affect the content, and all legal disclaimers that apply to the journal pertain. 
to refer to healthy volunteers from the community for testing. However, in practice, screening is often extended to refer to patients who may have presented to medical providers for other reasons for testing during medical encounters where no symptoms are present. The latter is sometimes referred to as case-detection or as case finding. Screening tests vary in content and could be comprised of questionnaires, a physical examination, and laboratory evaluation or imaging studies. (7)

Screening and diagnostic testing are distinguished by the role of patient symptoms in disease detection. While screening occurs among asymptomatic individuals, diagnostic testing occurs among individuals who have sought medical attention to identify a cause for a symptom.

Screening tests are often performed among large populations with the goal of identifying the most disease with a minimum level of associated cost. Thus, screening is often conducted using a test that, if positive, will need confirmation with a more definitive (and usually more costly) test. Because chronic kidney disease (CKD) in its earliest stages is asymptomatic, screening tests to identify $\mathrm{CKD}$ are largely laboratory-based and include determination of reduced kidney function[EF2] and/or kidney damage through the quantification of analytes such as creatinine from blood samples or protein levels in urine.

\section{Conditions Conducive to Screening}

When determining whether screening is worthwhile, several factors should be considered. First, and probably most important, is that the disease should be relatively common or prevalent among the population targeted for screening. Screening efforts are likely to be more fruitful and therefore less costly when the disease or condition of interest is relatively common. While there is no accepted threshold for a prevalence that triggers screening, screening is more controversial for conditions that are rare (e.g. less than 1 person detected per 100 persons screened). Second, a screening test must have good performance characteristics. These characteristics include sensitivity (the true-positive rate), specificity (1- the false-positive rate) and predictive value (proportion of persons who test positive that have the disease). Tests with optimal performance characteristics optimize chances for disease detection while minimizing the burden of false positives which can lead to unnecessary harm from testing and additional costs. Third, because all tests are not benign and have the possibility of inducing psychological [EF3](e.g. false labeling of persons with disease or true labeling with no viable strategy to help them) as well as physical harm, screening will be conducive when harms as a result of testing are minimal or do not exist. Fourth, the disease identified through screening must be able to be treated either for cure or to delay progression. Fifth, the benefits from a longer or better quality of life due to cure or delayed progression of disease must outweigh the harms of testing. Finally, circumstances under which screening costs are minimal will be viewed more favorably. Many of these considerations are captured in an analytic framework for evaluating screening evidence proposed by the United States Preventive Services Task Force (8) (Figure 1).

\section{Other Considerations in Screening Programs}

A variety of additional considerations contribute to the feasibility of implementing screening programs. If many tests are available for screening, the choice of one test for screening and the identification of specific criteria for further action (e.g. urine testing for proteinuria or microalbumiuria and sex specific cutoffs for microalbuminia vs a uniform threshold for men and women) represents one major consideration. A second consideration is the setting in which screening might be most effective (e.g. during periodic health examinations to primary care physicians, during physician visits addressing other problems, at the workplace, or in community settings such as health fairs). Third, the screening may be most advantageous in certain high risk groups; for example in those with risk factors such as hypertension, diabetes, obesity and African American race. Fourth, identification of an ideal screening frequency is 
very important (e.g. every few months, annually, or over multiples of years). More frequent testing could incur more cost and more harms. While the success of the first year of a screening program is determined by the prevalence rate of a disease, the utility of screening in subsequent years is determined by the incidence rate of a disease. Diseases with low incidence rates and sufficiently slow progression may require less frequent screening to identify cases at an acceptable rate. Fifth, testing induced by screening studies should be considered. For example, a positive dipstick for proteinuria would require subsequent quantitative testing of albuminuria, assessment of kidney function (via measurement of serum creatinine or cystatin-C) and other tests to rule out treatable causes for proteinuria (such as hepatitis) and perhaps a kidney biopsy. Finally, individuals identified with disease might have a choice of treatments and follow-up management.

\section{Is Screening for CKD Worthwhile?}

CKD has now been shown to exist in up to $13 \%$ of the general population (9), a prevalence that is comparable to and even greater than other chronic conditions (e.g. mild thyroid failure) for which screening may be useful. Research suggests that single measurements of dipstick proteinuria are strong predictors of end-stage renal disease (ESRD) in men, but in the United States general population-based screening does not appear to be cost-effective $(10,11)$. However, targeted screening to subgroups of the population might deliver the screening intervention to those who would derive the most benefit from CKD detection. (11)

Arguably the best evidence for screening would be a randomized screening trial performed in large number of individuals throughout the U.S. who are followed over many years (perhaps their lifetime) for outcomes. Such research has been performed for mammographic screening (11). However, such trials are difficult to execute and expensive to perform. The use of evidence and decision modeling to capture all components of the framework in Figure 1 is an alternative.

Based on evidence, de Jong and Curhan make the case that screening for albuminuria in high risk individuals fulfills criteria to initiate a screening program $(13,14)$. Another study more specifically addressed the question -- Is it cost effective to periodically screen adults, age 3070 years, in the US population for macro-albuminuria with a urine dipstick versus waiting for kidney disease to clinically emerge and be treated according to usual medical practice? (11) It also addressed whether there are subpopulations for whom screening is more cost effective (e.g. hypertensive patients). The study design employed cost-effectiveness analysis using Markov decision modeling, a technique in which patients are assumed to be in health states and make transitions to other healthy or diseased states over time. In this research, the intervention for screening was a periodic urine dipstick test performed annually. The screening population was adults, age 30-70 years, in the U.S. with neither hypertension nor diabetes or with hypertension. The cost-effectiveness analysis examined lifetime risks and took a societal perspective with regard to who is responsible for the cost of resources consumed as a result of screening or not screening. The outcomes were length and quality of life (measured in quality adjusted life years), costs due to medical transactions (direct costs) and costs due to lost wages and productivity (indirect medical costs). All these outcomes were tallied and expressed in a cost-effectiveness ratio incorporating the difference in costs between the screening strategy and usual care and the differences in length and quality of life between the screening strategy and usual care.

Figure 2 depicts the strategies and subsequent health states for individuals. Probabilities for transitioning between these health states were obtained from literature sources which employed the greatest methodologic rigor. Costs of screening, subsequent evaluation and treatment were obtained through available economic data. 
In the base case, in which screening was performed annually among persons age 50 years or older in the general population, the cost effectiveness ratio for screening persons with neither hypertension nor diabetes was $\$ 282,818$ per QALY (not cost-effective). In comparison, the cost-effectiveness ratio for screening persons with hypertension was \$18,621 per QALY (highly cost-effective), and a sensitivity analysis performed for comparisons with screening for persons with diabetes revealed screening for diabetes was both cost saving and saved lives. With regard to the harms of proteinuria screening, screening 1 million people with neither hypertension nor diabetes would result in 135 kidney biopsies, 7 kidney biopsy complications and complication costs of $\$ 9116$ per year. Screening 1 million people with hypertension would result in 196 kidney biopsies, 16 kidney biopsy complications and complication costs of $\$ 20,000$ per year.

In contrast to annual screening, screening persons age 50 years or older with neither hypertension nor diabetes at a frequency of every ten years approached favorable costeffectiveness ratios. Compared to annual screening for persons age 50 years or older, annual screening was more cost-effective in persons age 60 years or older and cost-effectiveness of screening improved with lengthening of the interval between screenings to more than annually (Figure 3). Figure 4 shows that annual screening was not cost-effective unless targeted at high risk groups such as the general population age 60 years or older and those with hypertension. The findings by Collins et al in this issue are consistent with this analysis (9).

\section{Comparison of CKD Screening with Other Screening Interventions}

To interpret the value of a new screening strategy, the cost-effectiveness is often compared with screening programs for other conditions. Screening for CKD with urine protein testing among persons age 50 years or older with neither diabetes nor hypertension on an annual basis is very favorable ( $<\$ 50,000$ per quality adjusted life year) and similar to screening for cervical cancer, for abdominal aortic aneurysm, and for type 2 diabetes among persons with hypertension (Figure 4). Screening persons age 60 with neither diabetes nor hypertension on an annual basis is moderately cost-effective as is screening persons age 50 in the general population every 10 years.

\section{Factors that Influence the Value of Screening for CKD}

Sensitivity analyses revealed the factors largely driving the cost-effectiveness of screening. These factors include patient and provider adherence to medications which modulate the reninangiotensin-aldosterone system (angiotensin-converting enzyme inhibitors or angiotensin reception blockers), the reduction in death from such therapy, the incidence of between intervals of screening[EF4], and quality of life due to side effects of therapy. Sensitivity and specificity of the urine dipstick screening for detection of proteinuria have only a modest effect. Other factors, not examined to date, which also may affect the value of screening are targeting to other high risk populations (e.g. persons with African American race, obesity, smokers, and other CVD risk factors).

\section{Global Experiences with Population Screening for CKD}

Developing screening programs among nations with different health care considerations is challenging (1). In 2004, an International Society of Nephrology Consensus Workshop on Prevention of Progression of Renal Disease recommended that patients with diabetes, hypertension and relatives with kidney disease should have regular screening for development of kidney disease (2). More recently, in 2006, experts from around the world examined CKD as a global public health problem addressing the issue of surveillance through the Kidney Disease Improving Global Outcomes (KDIGO) initiative (3). Experts from different countries made several recommendations with respect to CKD screening (Box 1). First, they stated that 
all countries should have a targeted screening program for CKD with target groups including patients with hypertension, diabetes and cardiovascular disease. Depending on local circumstances other groups to be included might be families of patients with CKD, individuals with hyperlipidemia, obesity, metabolic syndrome, smokers, patients treated with potentially nephrotoxic drugs, some chronic infectious diseases and cancers, and age $>60$ years. Second, they stated that the tests for CKD screening should include both a urine test for proteinuria with follow up with a blood test for creatinine to estimate glomerular filtration rate (GFR). Tests for proteinuria should be selected and performed according to local guidelines and verification of proteinuria would require two out of three positive tests. Equations for estimating GFR should be appropriate for standardization of the method and application to majority racial and ethnic groups. With regard to frequency of testing, groups recommended available guidelines and target groups should be tested, but in the absence of specific recommendations, testing need not be more frequent than once per year.

In 2007 a United Kingdom Consensus Conference on Early Chronic Kidney Disease proclaimed that there is a lack of evidence to support the cost effectiveness of general population screening and that the majority of cases will be detected from blood sampling and chronic disease management clinics in primary care using eGFR. (4)

A National Kidney Foundation position statement supports early detection of CKD, but recommends only asymptomatic individuals at increased risk be tested with explicit testing strategies (5)

In Japan, urine testing in annual health examinations has been credited with reducing the number of patients with ESRD due to glomerulonephritis. Based on this experience, screening programs have been recommended to target patients with diabetes, hypertension, and the metabolic syndrome (6).

While it is clear worldwide consensus is building regarding the need for targeted screening programs, variation in recommendations may reflect lack of evidence to guide specific aspects of program implementation and uncertainty regarding the cost-quality tradeoffs associated with different screening strategies. For example, recommendations requiring two out of three positive tests may reflect concern regarding the specificity of inexpensive tests which could be used to screen CKD. Quantitative tests for urine protein are more accurate but are also more expensive. In addition, it is unclear whether screening for CKD should be performed in a standalone fashion or in combination with other well-established screening programs such as programs for screening cholesterol and high blood pressure. Future work to establish evidence upon which details regarding screening programs can be based is needed.

\section{Recognition and Management of CKD in Clinical Care}

If screening is not cost-effective in the general population, it is important to consider whether our health care system is equipped to recognize early stages of CKD that emerge in the course of routine clinical care of patients. Research examining physician recognition of persons with CKD addresses this issue. A study of primary care physicians and nephrologists demonstrated primary care physicians were less likely than nephrologists to correctly identify patients with Stage 3-4 CKD and less likely to recommend referral to nephrologists in accordance with recent clinical practice guidelines (15). Physicians in practice more than 10 years were less likely to identify patients with stage 3-4 CKD. Furthermore, generalists and specialists disagreed on the diagnostic evaluation for patients with CKD. In another study, primary care physicians were less likely to recognize African American race and family history of ESRD as risk factors for CKD progression (16). Furthermore, in a study at training institutions, CKD risk factors were not recognized, screening for CKD complications was not generally taken into consideration, and management of anemia and secondary hyperparathyroidism was 
difficult for both family medicine and internal medicine trainees and attending physicians

(17). Tools for screening and recognition may be useful in identifying persons at risk for CKD

(18). Thus, regardless of whether CKD is addressed through screening or routine care, more

education and more collaboration between primary care physicians and specialists is needed.

\section{Conclusion}

Screening for chronic diseases such as CKD can be valuable if applied appropriately. Much of the evidence regarding population based screening for CKD suggests that screening should be targeted, rather than universally performed, and screening should be performed with urine testing followed by creatinine-based eGFR testing. The value of screening may be enhanced by implementing it along with other disease detection programs used in the general population. Future work should continue to examine the performance characteristics of different screening tests in various settings, optimal timing intervals for screening and the benefits, risks and costs of screening strategies with various components. This will help address global consensus on approaches and recommendations for screening. In addition, research on new biomarkers for kidney disease identification that can be used in screening and research on new pharmacologic agents for more effective protection from kidney disease damage or progression should be supported.

\section{Box 1: Recommendation on Population Screening for CKD from the Kidney Disease Improving Global Outcomes conference in Amsterdam 2006. From [3]}

- All countries should have a targeted screening program for CKD.

- Target groups should include patients with hypertension, diabetes and cardiovascular disease.

- Other groups might include families of patients with CKD, individuals with hyperlipidemia, obesity, metabolic syndrome, smokers, patients treated with potentially nephrotoxic drugs, some chronic infectious diseases and cancers, and age $>60$ years.

- Tests for CKD screening should include both a urine test for proteinuria and a blood test for creatinine to estimate GFR.

- Tests for proteinuria should be selected and performed according to local guidelines. Verification of proteinuria would require two out of three positive tests.

- Equations for estimating GFR should be appropriate for standardization of the method and application to majority racial and ethnic groups.

- Frequency of testing should be according to available guidelines and target group to be tested. In absence of specific recommendations, testing need not be more frequent than once per year.

\section{Acknowledgements}

Support: Dr Powe is supported by grant K240502643 from the National Institute of Diabetes and Digestive and Kidney Diseases and Dr Boulware by grant K23DK070757 from the National Center for Minority Health and Health Disparities and the National Institute of Diabetes and Digestive and Kidney Diseases. 


\section{References}

1. Codreanu I, Perico N, Sharma SK, et al. Prevention programmes of progressive renal disease in developing nations. Nephrology 2006;11:321-328. [PubMed: 16889572]

2. Li, PK.; Weening, JJ.; Dirks, J.; Lui, SL.; Szeto, CC.; Tang, S.; Atkins, RC.; Mitch, WE.; Chow, KM.; D’Amico, G.; Freedman, BI.; Harris, DC.; Hooi, LS.; Jong, PE.; Kincaid-Smith, P.; Lai, KN.; Lee, E.; Li, FK.; Lin, SY.; Lo, WK.; Mani, MK.; Mathew, T.; Murakami, M.; Qian, JQ.; Ramirez, S.; Reiser, T.; Tomino, Y.; Tong, MK.; Tsang, WK.; Tungsanga, K.; Wang, H.; Wong, AK.; Wong, KM.; Yang, WC.; Zeeuw, D.; Yu, AW.; Remuzzi, G. Participants of ISN Consensus Workshop on Prevention of Progression of Renal Disease. Kidney Int Suppl; A report with consensus statements of the International Society of Nephrology 2004 Consensus Workshop on Prevention of Progression of Renal Disease; Hong Kong. June 29, 2004; 2005 Apr. p. S2-7.

3. Levey AS, Atkins R, Coresh J, Cohen EP, Collins AJ, Eckardt KU, Nahas ME, Jaber BL, Jadoul M, Levin A, Powe NR, Rossert J, Wheeler DC, Lameire N, Eknoyan G. Chronic kidney disease as a global public health problem: approaches and initiatives - a position statement from Kidney Disease Improving Global Outcomes. Kidney Int 2007 Aug;72(3):247-59. [PubMed: 17568785]

4. Archibald G, Bartlett W, Brown A, Christie B, Elliott A, Griffith K, Pound S, Rappaport I, Robertson D, Semple Y, Slane P, Whitworth C, Williams B. UK Consensus Conference on Early Chronic Kidney Disease. Nephrol Dial Transplant 2007;22(Suppl 9):ix4-ix5.(Chair)

5. Vassalotti JA, Stevens LA, Levey AS. Testing for chronic kidney disease: a position statement from the National Kidney Foundation. Am J Kidney Dis 2007;50(2):169-80. [PubMed: 17660017]

6. Imai E, Yamagata K, Iseki K, et al. Kidney disease screening program in Japan: History, outcome, and perspectives. Clinical Journal of the American Society of Nephrology 2:1360-1366.200

7. Sackett DL, Holland WW. Controversy in the detection of disease. Lancet 1975 Aug 23;2(7930):3579. [PubMed: 51154]

8. Harris, et al. Current methods of the US Preventive Services Task Force: A review of the process. Am J Prev Med 2001;20(3S):21-35. [PubMed: 11306229]

9. Coresh J, Selvin E, Stevens LA, Manzi J, Kusek JW, Eggers P, Van Lente F, Levey AS. Prevalence of chronic kidney disease in the United States. JAMA 2007;298(17):2038-47. [PubMed: 17986697]

10. Ishani A, Grandits GA, Grimm RH, Svendsen KH, Collins AJ, Prineas RJ, Neaton JD. for the MRFIT Research Group. Association of single measurements of dipstick proteinuria, estimated glomerular filtration rate, and hematocrit with 25-year incidence of end-stage renal disease in the Multiple Risk Factor Intervention Trial. J Am Soc Nephrol 2006;17:1444-1452. [PubMed: 16611715]

11. Boulware LE, Jaar BG, Tarver-Carr ME, Brancati FL, Powe NR. Screening for Proteinuria in US Adults: A Cost-effectiveness Analysis. JAMA 2003;290:3101-3114. [PubMed: 14679273]

12. Olsen, O.; Gøtzsche, PC. Cochrane Database Syst Rev. 2001. Screening for breast cancer with mammography; p. CD001877Review. Update in: Cochrane Database Syst Rev. 2006

13. Wilson, JMG.; Jungner, F. Public Health Papers, No 34. Geneva: World Health Organization; 1968. Principles and Practice of Screening for Disease.

14. de Jong PE, Curhan GC. Screening, monitoring, and treatment of albuminuria: Public health perspectives. Journal of the American Society of Nephrology 2006;17:2120-126. [PubMed: 16825331]

15. Boulware LE, Troll MU, Jaar BG, Myers DI, Powe NR. Identification and referral of patients with progressive CKD: a national study. Am J Kidney Dis 2006;48:192-204. [PubMed: 16860184]

16. Lea JP, McClellan WM, Melcher C, Gladstone E, Hostetter T. CKD risk factors reported by primary care physicians: do guidelines make a difference? Am J Kidney Dis 2006;47:72-77. [PubMed: 16377387]

17. Lenz O, Fornoni A. Chronic kidney disease care delivered by US family medicine and internal medicine trainees: results from an online survey. BMC Med 2006;4:30. [PubMed: 17164005]

18. Bang H, Vupputuri S, Shoham DA, Klemmer PJ, Falk RJ, Mazumdar M, Gipson D, Colindres RE, Kshirsagar AV. SCreening for Occult REnal Disease (SCORED): a simple prediction model for chronic kidney disease. Arch Intern Med 2007;167:374-81. [PubMed: 17325299] 
Screening

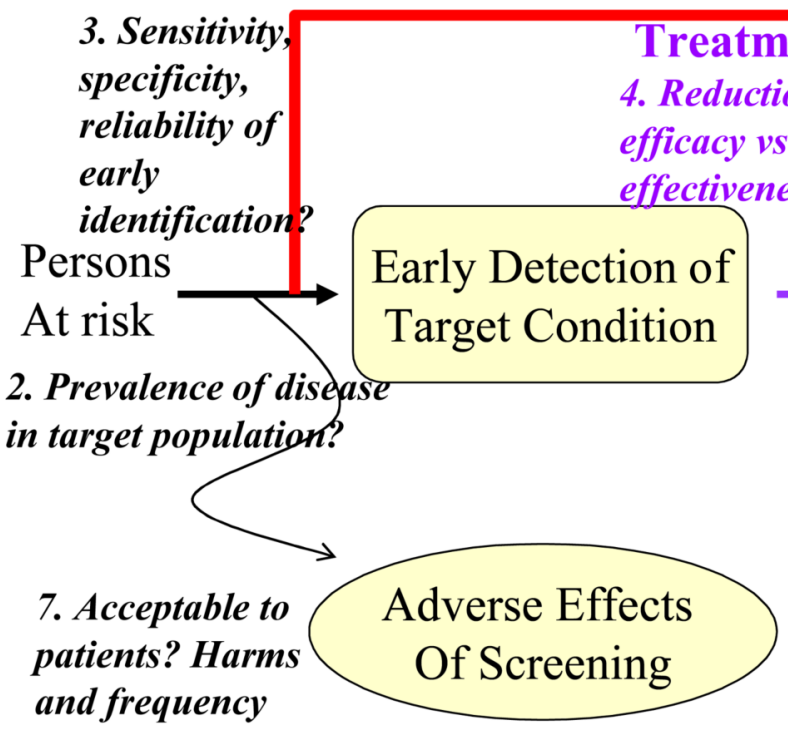

1. Direct evidence?

\section{ment}

s.

5. Does treatment improve patient outcomes?
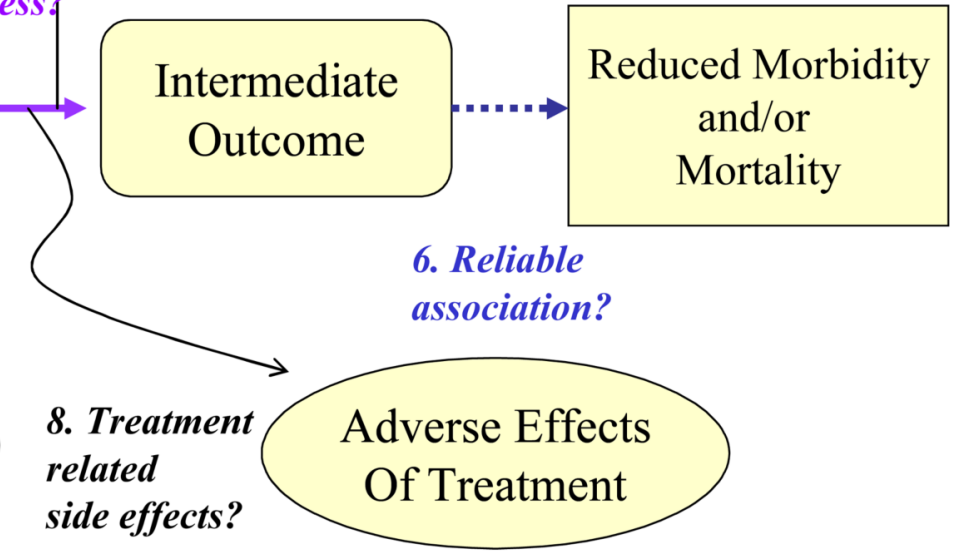

Figure 1.

Analytic Framework for Evidence on Screening Topics from the United States Preventive Services Taskforce. From [8]. 
Progressien Slewed By ACE Inhibitor or AAB Therapy

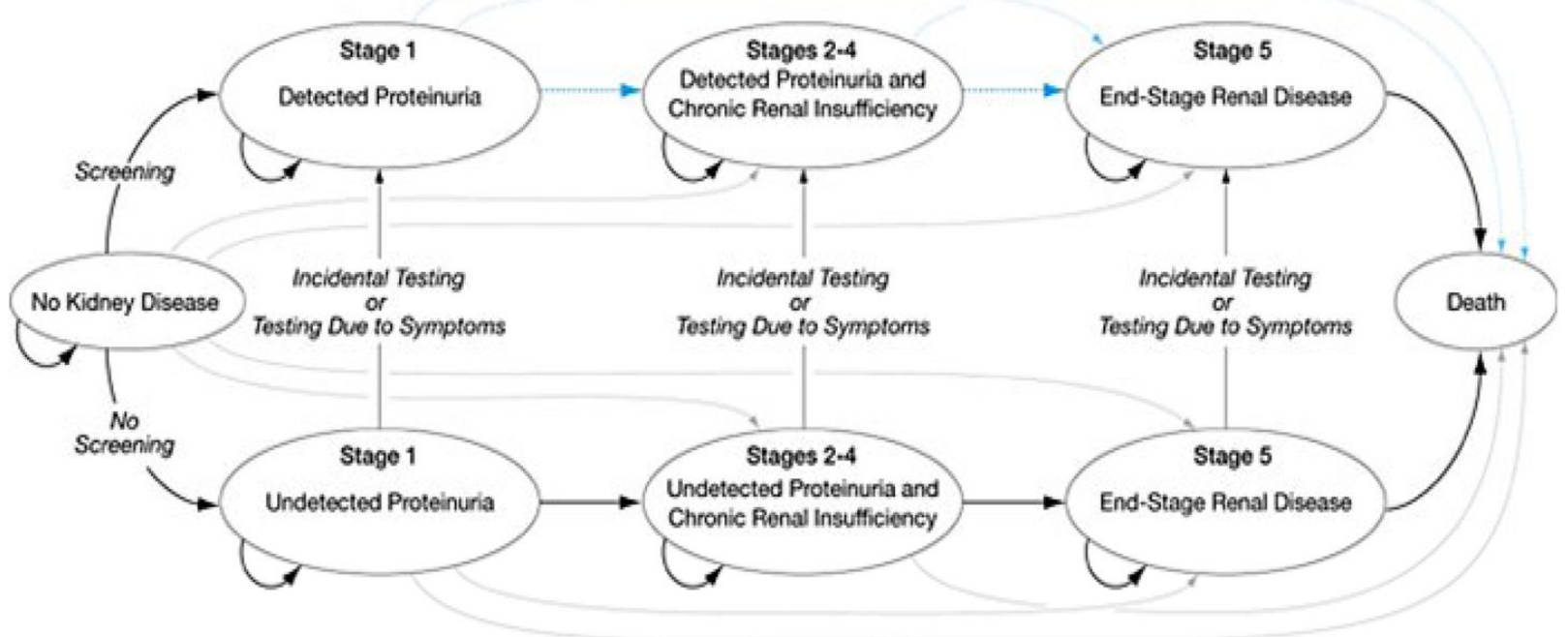

Figure 2.

Depiction of Screening Strategies, Health States and Possible Transitions in Decision Analytic Model Comparing the Cost-effectiveness of Screening for Proteinuria to Identify Chronic Kidney Disease. From [11]. 


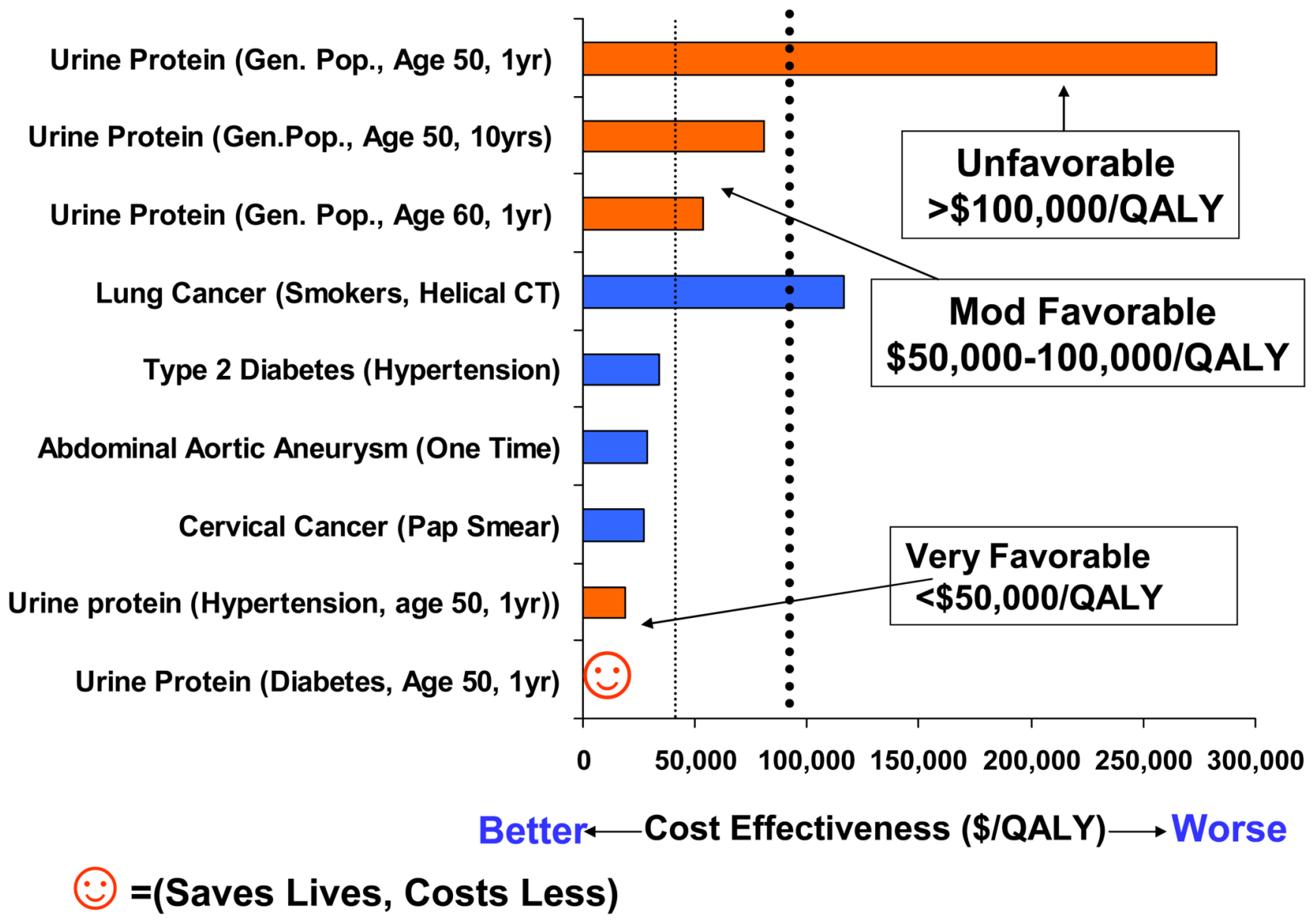

Figure 3.

Comparison of the Cost-effectiveness of Screening for Chronic Kidney Disease with urine dipstick testing with screening intervention for other diseases. Adapted from [11]. 


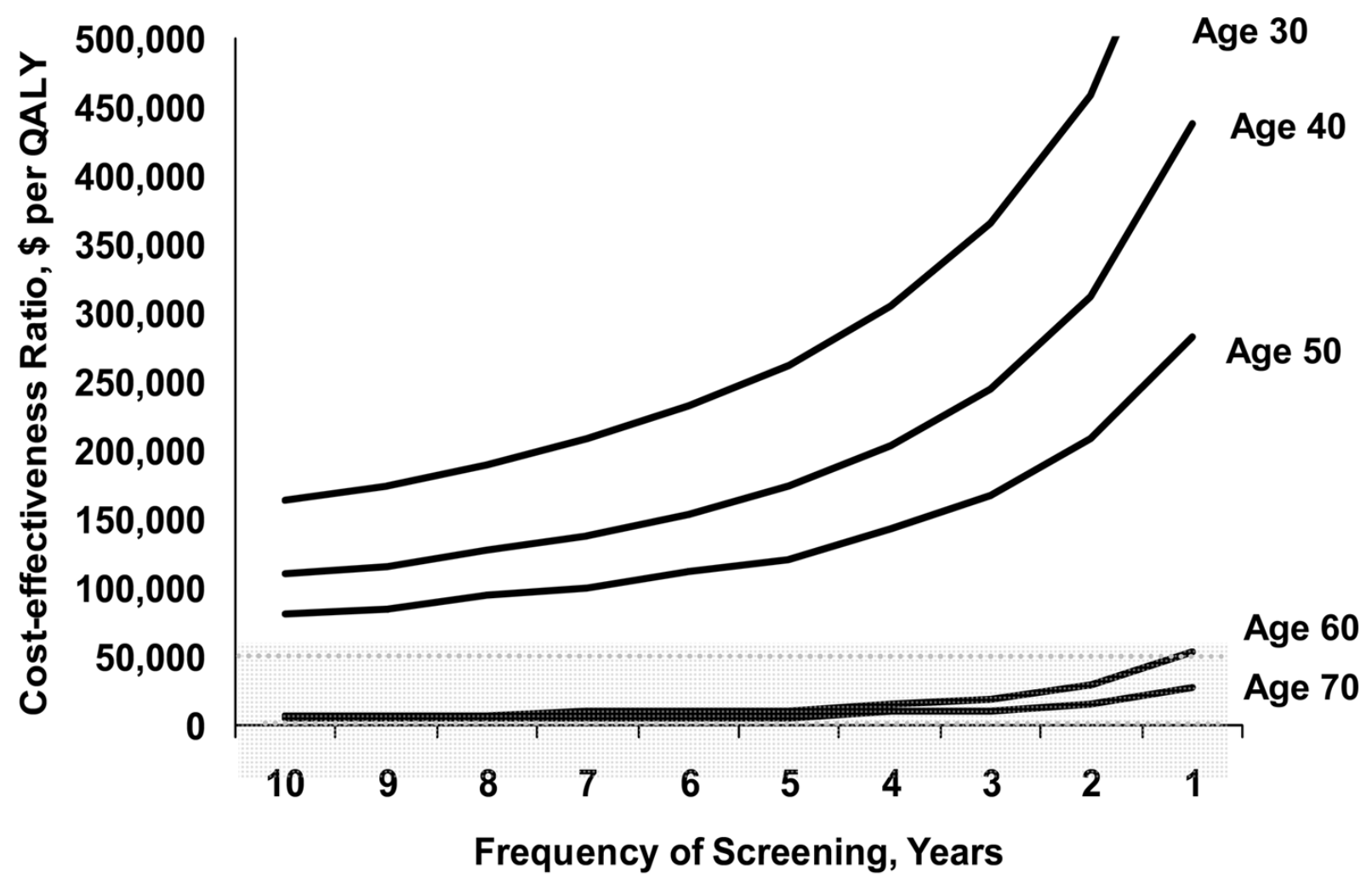

Figure 4.

Cost-effectiveness of Screening for Proteinuria at Different Frequencies and Ages for Persons with Neither Hypertension nor Diabetes. From [11]. 